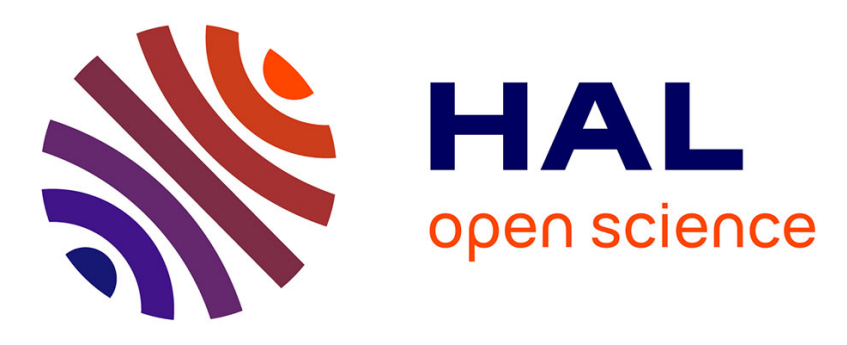

\title{
Negatively Charged Excitons in CdSe Nanoplatelets
}

Elena V Shornikova, Dmitri R Yakovlev, Louis Biadala, Scott A Crooker, Vasilii V Belykh, Mikhail V Kochiev, Alexis Kuntzmann, Michel Nasilowski, Benoit Dubertret, Manfred Bayer

\section{- To cite this version:}

Elena V Shornikova, Dmitri R Yakovlev, Louis Biadala, Scott A Crooker, Vasilii V Belykh, et al.. Negatively Charged Excitons in CdSe Nanoplatelets. Nano Letters, 2019, 20 (2), pp.1370-1377. 10.1021/acs.nanolett.9b04907 . hal-03000677

\section{HAL Id: hal-03000677 https://hal.science/hal-03000677}

Submitted on 12 Nov 2020

HAL is a multi-disciplinary open access archive for the deposit and dissemination of scientific research documents, whether they are published or not. The documents may come from teaching and research institutions in France or abroad, or from public or private research centers.
L'archive ouverte pluridisciplinaire HAL, est destinée au dépôt et à la diffusion de documents scientifiques de niveau recherche, publiés ou non, émanant des établissements d'enseignement et de recherche français ou étrangers, des laboratoires publics ou privés. 


\title{
Negatively charged excitons in CdSe nanoplatelets
}

\author{
Elena V. Shornikova, ${ }^{*} \dagger$ Dmitri R. Yakovlev,,,++ Louis Biadala, 9 Scott A. \\ Crooker, ${ }^{\S}$ Vasilii V. Belykh," Mikhail V. Kochiev, $\|$ Alexis Kuntzmann, ${ }^{\perp}$ Michel \\ Nasilowski, ${ }^{\perp}$ Benoit Dubertret, ${ }^{\perp}$ and Manfred Bayer ${ }^{\dagger, \ddagger}$ \\ †Experimentelle Physik 2, Technische Universität Dortmund, 44221 Dortmund, Germany \\ $\ddagger$ Ioffe Institute, Russian Academy of Sciences, 194021 St. Petersburg, Russia \\ ฯInstitut d'Electronique, de Microélectronique et de Nanotechnologie, CNRS, 59652 \\ Villeneuve-d'Ascq, France \\ $\S$ National High Magnetic Field Laboratory, Los Alamos National Laboratory, Los Alamos, \\ 87545 New Mexico, United States \\ \|P. N. Lebedev Physical Institute, Russian Academy of Sciences, 119991 Moscow, Russia \\ $\perp$ Laboratoire de Physique et d'Etude des Matériaux, ESPCI, CNRS, 75231 Paris, France \\ E-mail: elena.shornikova@tu-dortmund.de; dmitri.yakovlev@tu-dortmund.de
}

\section{Abstract}

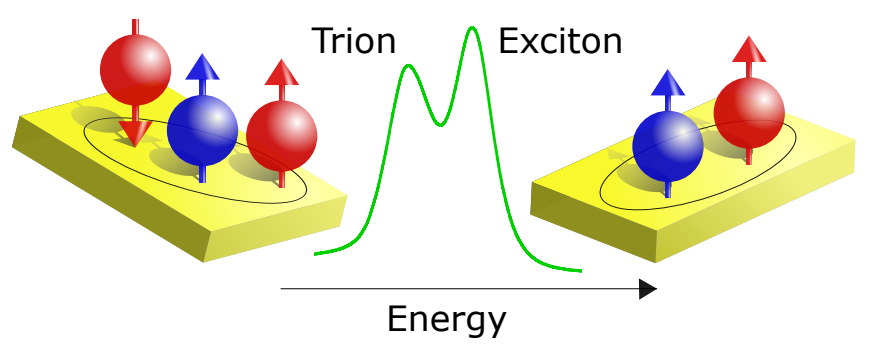

The low-temperature emission spectrum of CdSe colloidal nanoplatelets (NPLs) consists of two narrow lines. The high-energy line stems from the recombination of neutral excitons. The origin of the low-energy line is currently debated. We experimentally study the spectral shift, emission dynamics, and spin polarization of both lines at low temperatures down to $1.5 \mathrm{~K}$ and in high magnetic fields up to $60 \mathrm{~T}$, and show that the low energy line originates from the recombination of negatively charged excitons (trions). This assignment is confirmed by the NPL photocharging dynamics and associated variations in the spectrum. We show that the negatively charged excitons are considerably less sensitive to the presence of surface spins than the neutral excitons. The trion binding energy in 3-monolayer-thick NPLs is as large as $30 \mathrm{meV}$, which is four times larger than its value in two-dimensional limit of a conventional CdSe quantum well confined between semiconductor barriers. A considerable part of this enhancement is gained by the dielectric enhancement effect, that is due to the small dielectric constant of the environment surrounding the NPLs.

Keywords: Colloidal nanocrystal, CdSe nanoplatelet, charged exciton, trion, high magnetic fields.

The optical properties of colloidal nanostructures depend strongly on their shape. In recent years there has been increasing interest in colloidal nanoplatelets (NPLs), which are atomically flat, two dimensional semiconductor layers with several monolayers thickness that resemble free-standing quantum wells and exhibit excellent optical properties. ${ }^{1,2}$ Nanoplatelets made of CdSe show spectrally narrow ensemble emission and short spontaneous recombination times, which potentially make them among the most efficient colloidal luminophores. ${ }^{3}$

An electron-hole pair photogenerated in a 
high quality nanostructure usually recombines by emitting a photon. Alternatively, one of the photoexcited carriers can escape from the nanostructure and become trapped by surface states or traps in the surrounding material, as observed for spherical CdS and CdSe nanocrystals (NCs). ${ }^{4,5}$ Thereby the nanostructure becomes charged by the remaining electron or hole. In some cases, both the electron and hole might be trapped on the surface from which they subsequently return back to the nanostructure after different detrapping times. ${ }^{4,6}$ Photoexcitation of singly-charged nanostructures generates charged excitons, also known as trions because of their composition of three carriers. Trions can be either negatively charged (consisting of two electrons and one hole) or positively charged (composed of one electron and two holes).

Negatively and positively charged trions have been intensively studied in epitaxially grown quantum well (QW) structures since 1993. ${ }^{7}$ Comprehensive information on their properties, including their spin, in QWs based on GaAs, CdTe, ZnSe etc. has been accumulated, largely by using magneto-optical spectroscopy at cryogenic temperatures. In small colloidal NCs the trion photoluminescence (PL) is completely suppressed by the efficient nonradiative Auger recombination. ${ }^{8}$ Trion PL was observed in colloidal CdSe/CdS NCs ${ }^{9-12}$ and dot-in-bulk structures, ${ }^{13}$ where Auger processes are suppressed by the thick shell. ${ }^{14-17}$ In bare CdSe NPLs the nonradiative Auger recombination time of biexcitons was measured to be on the order of $0.25 \mathrm{~ns},{ }^{18}$ which is significantly longer than in CdSe NCs. This suggests that Auger recombination time of trions in CdSe NPLs is on the order of $0.5 \mathrm{~ns}$, which is sufficiently long to allow observation of trion band-edge PL from NPLs.

The interpretation of the band-edge emission in CdSe NPLs is controversial. Their PL spectrum at cryogenic temperatures typically consists of two well-resolved lines. The highenergy line was proven to arise from neutral exciton recombination, ${ }^{19,20}$ while the origin of the low-energy line is still actively debated. Among the considered options are LO-phonon assisted exciton recombination, ${ }^{21}$ trion emission, ${ }^{21}$ emission from excimer states in neighboring NPLs, ${ }^{22}$ and recombination from ground $s$ - exciton state. ${ }^{23}$ In our recent paper on the fine structure of neutral excitons in CdSe NPLs $^{20}$ we presented arguments in favor of the trion interpretation of the low-energy line. However, this was a side aspect of that paper. Here we present a systematic in-depth study of the low-energy PL line in CdSe NPLs.

There are several experimental approaches that can be used to identify and distinguish between exciton and trion emission in colloidal NCs:

(i) The recombination dynamics of excitons and trions are typically quite different. The exciton dynamics are strongly modified by temperature and magnetic field due to mixing of the groundstate, which is optically inactive (dark) excitons, with higher-lying, optically active (bright) exciton states. In contrast, the trions are always optically active so that the trion $\mathrm{PL}$ is insensitive to these factors.

(ii) The PL polarization in external magnetic field is controlled by the spin state of the exciton complex. For neutral excitons it is determined by its $g$-factor, $g_{\mathrm{X}}$, to which the electron $\left(g_{\mathrm{e}}\right)$ and hole $\left(g_{\mathrm{h}}\right) g$-factors contribute. In contrast, the Zeeman splitting of trions is given only by $g_{\mathrm{h}}$ for negatively charged trions or by $g_{\mathrm{e}}$ for positively charged trions. Note that the optical transition of the trions split with $g_{\mathrm{X}}$, as they are contributed also by the Zeeman splitting of the resident carrier left after trion recombination.

(iii) The different spin structure of excitons and trions can lead to different interactions with surface spins, which can be detected via polarized emission. ${ }^{24}$

(iv) Because the formation of trions necessarily requires the presence of resident charge carriers, photocharging provides an additional tool to identify the presence of trion states. The efficiency and time evolution of photocharging can be studied by a variety of experimental techniques, including PL spectroscopy, pump-probe Faraday rotation, ${ }^{4,5}$ or spin-flip Raman scattering. ${ }^{25}$

(v) The spectral shift of the low-energy PL line 
can help to distinguish between trion emission and LO-phonon assisted recombination of dark excitons. One can analyze the spectral shifts as a function of NPL thickness and compare them with LO phonon energies. Note that the LO phonon energies are only weakly dependent on the NPL thickness, while the exciton and trion energies increase strongly with decreasing NPL thickness due to quantum confinement and dielectric enhancement.

(vi) In single-NC measurements, the exciton and trion PL lines exhibit different Zeeman splittings and polarizations in magnetic field. Also, they obviously have to show an anti-correlated behavior: only exciton emission is possible from neutral NCs, while only trion emission is possible from singly-charged NCs. ${ }^{26-28}$

(vii) In has been shown recently that the low-temperature emission spectra of individual CdSe/CdS NPLs may contain several emission lines, which are identified as recombination of the negatively charged excitons accompanied by a shakeup process of the resident electron. ${ }^{12}$

In this paper we exploit several of these approaches to study ensembles of bare-core CdSe NPLs at cryogenic temperatures (down to $1.5 \mathrm{~K}$ ) and in high magnetic fields up to $60 \mathrm{~T}$. We confirm that the low-energy emission line in the studied CdSe NPLs originates from negatively charged trions.

We study bare-core CdSe NPLs with thicknesses of 3, 4 and 5 monolayers (MLs). Some information on their optical properties, exciton parameters, and spin-dependent properties can be found in Refs. 20,24,25. Here, we focus on the origin of the low-energy emission line and use the 5ML sample for presentation of results collected with the various experimental techniques. The results for the other samples are given in Supporting Information.

Photoluminescence and absorption spectra. Figure 1a shows the photoluminescence (PL) spectrum (red) measured at $T=4.2 \mathrm{~K}$, which consists of two lines with the high-energy line (X) at $2.319 \mathrm{eV}$ attributed to the exciton. ${ }^{20}$ The absorption (blue line) and PL excitation (PLE) (violet squares, detected at $2.28 \mathrm{eV}$ ) spectra coincide well. ${ }^{21}$ They have a maximum at $2.332 \mathrm{eV}$ from which the exciton emission is Stokes shifted by $13 \mathrm{meV}$. The low-energy emission line $(\mathrm{T})$ is shifted by $18 \mathrm{meV}$ to lower energies relative to the exciton. The X-T energy difference increases in the thinner NPLs: $20 \mathrm{meV}$ in the 4ML and $30 \mathrm{meV}$ in the 3ML NPLs (see Figure 1c and Supporting Information Figure S1). Note, that the excellent agreement between the PLE and absorption spectra highlights that both peaks in the PL spectrum originate from the same NPL population.

Recombination dynamics. One can distinguish excitons from trions by their different fine structure (Figure 1b). The ground (dark) exciton state $|F\rangle$ has net angular momentum projection \pm 2 onto the quantization axis, and is optically forbidden in the electricdipole approximation. The bright exciton state $|A\rangle$ with angular momentum projection \pm 1 is split from $|F\rangle$ by the bright-dark energy splitting, $\Delta E_{\mathrm{AF}}$, which increases from 3.3 to $6 \mathrm{meV}$ going from the $5 \mathrm{ML}$ to the $3 \mathrm{ML}$ thick NPLs. ${ }^{20}$ This exchange splitting determines the exciton PL dynamics at low temperatures when $k_{\mathrm{B}} T \ll \Delta E_{\mathrm{AF}}$, with $k_{\mathrm{B}}$ being the Boltzmann constant: the fast initial decay on a timescale of $30 \mathrm{ps}$ due to relaxation from the bright to the dark state is followed by a slow $\left(\tau_{\mathrm{F}}=58 \mathrm{~ns}\right)$ decay due to the nominally-forbidden dark state recombination (Figure 2b, pink).

In contrast, the trion ground state does not exhibit any fine structure splitting, because the two identical particles (e.g. the electrons in a negative trion) form a spin-singlet state. Radiative recombination is allowed for this spin configuration and therefore, the ground state of a trion is always bright ${ }^{29}$ (Figure $1 b$, bottom). Our measurements demonstrate that the lowenergy line exhibits a multi-exponential decay, shown by the green line in Figure 2b, and the black dashed line shows the corresponding fit. The initial fast component with $\tau_{\mathrm{T}}=450 \mathrm{ps}$ covers about two decades of the signal amplitude decay. We assign it to the trion radiative recombination. It lasts longer than the exciton initial decay (see inset Figure 2b), but much shorter than the dark exciton radiative decay $\tau_{\mathrm{F}}=\Gamma_{\mathrm{F}}^{-1}=58 \mathrm{~ns}^{20}$ The two further trion 

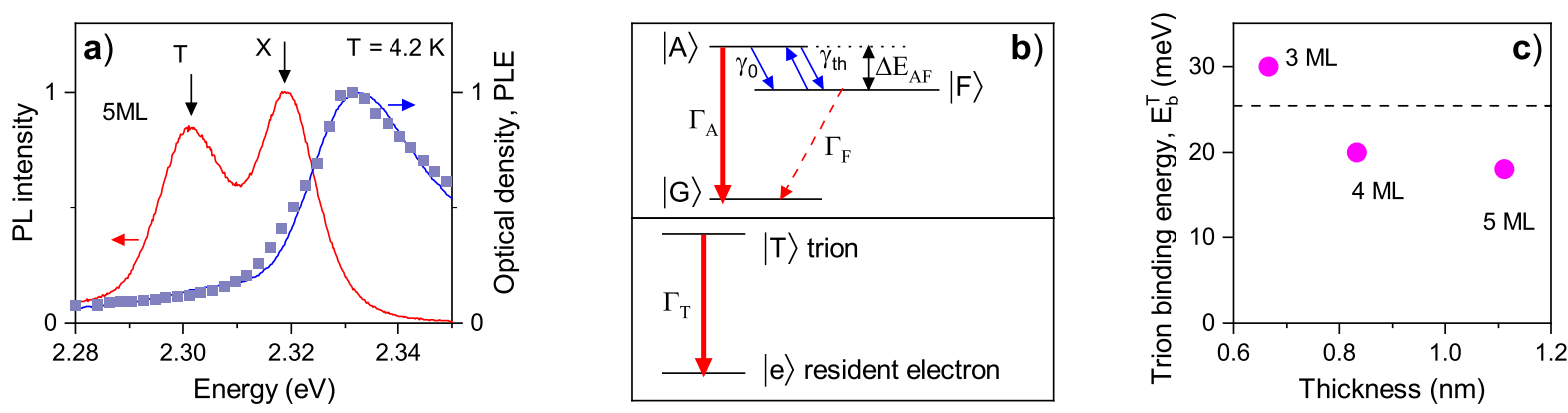

Figure 1: Photoluminescence, absorption, and PLE spectra. (a) Photoluminescence (red), absorption (blue), and PLE (violet squares) spectra of 5ML NPLs measured at $T=4.2 \mathrm{~K}$. The exciton $(\mathrm{X})$ and trion $(\mathrm{T})$ lines in emission are marked by arrows. (b) Top: Exciton fine structure: $|A\rangle$ and $|F\rangle$ are the bright and dark exciton states, $|G\rangle$ is the unexcited crystal ground state. $\Gamma_{\mathrm{A}}$ and $\Gamma_{\mathrm{F}}$ are the bright and dark exciton recombination rates, $\gamma_{0}$ is the relaxation rate from the bright to the dark state, and $\gamma_{\mathrm{th}}=\gamma_{0} N_{\mathrm{B}}$ is the thermal activation rate of the reverse process, where $N_{\mathrm{B}}=1 /\left[\exp \left(\Delta E_{\mathrm{AF}} / k_{\mathrm{B}} T\right)-1\right]$ is the Bose-Einstein phonon occupation. Bottom: Trion level structure: $|T\rangle$ is the negatively charged trion and $|e\rangle$ is the resident electron left after trion recombination. $\Gamma_{\mathrm{T}}=\tau_{\mathrm{T}}^{-1}$ is the trion recombination rate. (c) Splitting between the exciton (X) and low-energy $(\mathrm{T})$ lines, equal to the trion binding energy. The LO-phonon energy of $25.4 \mathrm{meV}$ is marked by the dashed line.

components with small amplitudes have decay times of $3 \mathrm{~ns}$ and $20 \mathrm{~ns}$. Most probably they are related to delayed formation of a fraction of trions from initially spatially separated resident electron and exciton within the same NPL.

\section{Recombination in external magnetic} fields. Figure 2c shows the PL dynamics of the low-energy line measured at zero magnetic field (green) and at $B=15 \mathrm{~T}$ (orange). While the decay rate of dark excitons typically accelerates in an external magnetic field due to the mixing of bright and dark states ${ }^{20,24}$ (not shown here), we find that the decay rate of the low-energy line remains unchanged. This behavior is typical for trion emission in colloidal NCs. ${ }^{10,13}$

Temperature-dependent time-resolved PL. Figure 2d shows the PL decays of the exciton and trion at various temperatures from 2 up to $50 \mathrm{~K}$. The signals were measured at the line maxima and integrated across the spectral ranges of $2.321-2.323 \mathrm{eV}$ and $2.298-2.300 \mathrm{eV}$, respectively. Increasing the temperature up to $50 \mathrm{~K}$ has no effect on the PL dynamics of the low-energy line (bottom panel), as expected for trion recombination. ${ }^{11}$ In contrast, the exciton line exhibits the typical temperaturedependent behavior (top panel): the bright ex- citon $|A\rangle$ level is populated at higher temperatures, which converts the two-exponential decay into a single-exponential decay with the overall rate $\left(\Gamma_{\mathrm{A}}+\Gamma_{\mathrm{F}}\right) / 2 \approx \Gamma_{\mathrm{A}} / 2$, where $\tau_{\mathrm{A}}=\Gamma_{\mathrm{A}}^{-1}=$ $100 \mathrm{ps}$ is the bright exciton radiative recombination time ${ }^{20}$ and $\Gamma_{\mathrm{F}} \ll \Gamma_{\mathrm{A}}$. One sees in Figure $2 \mathrm{~d}$ (top panel) that with increasing temperature the longer component decays faster while the fast component decays slower.

Degree of circular polarization (DCP). Excitons and trions differ in their spin level structure and as a result in their polarized emission in an external magnetic field. One can exploit this difference to identify the origin of the emission lines and also to distinguish negative and positive trions. This was demonstrated for CdSe-based spherical quantum dots ${ }^{10}$ as well as for NPLs. ${ }^{11,24}$

The PL spectrum at $B=15 \mathrm{~T}$ is shown in Figure 3a. The intensities of the right-handed $\left(\sigma^{+}\right)$and left-handed $\left(\sigma^{-}\right)$polarized components, $I^{+}$and $I^{-}$, are shown by the red and blue lines, respectively. The $\sigma^{-}$polarized emission dominates across the whole spectrum, so that the DCP, $P_{\mathrm{c}}=\left(I^{+}-I^{-}\right) /\left(I^{+}+I^{-}\right)$, is negative (cyan crosses). The DCP arises from the Zeeman effect, i.e. the splitting of the spin 

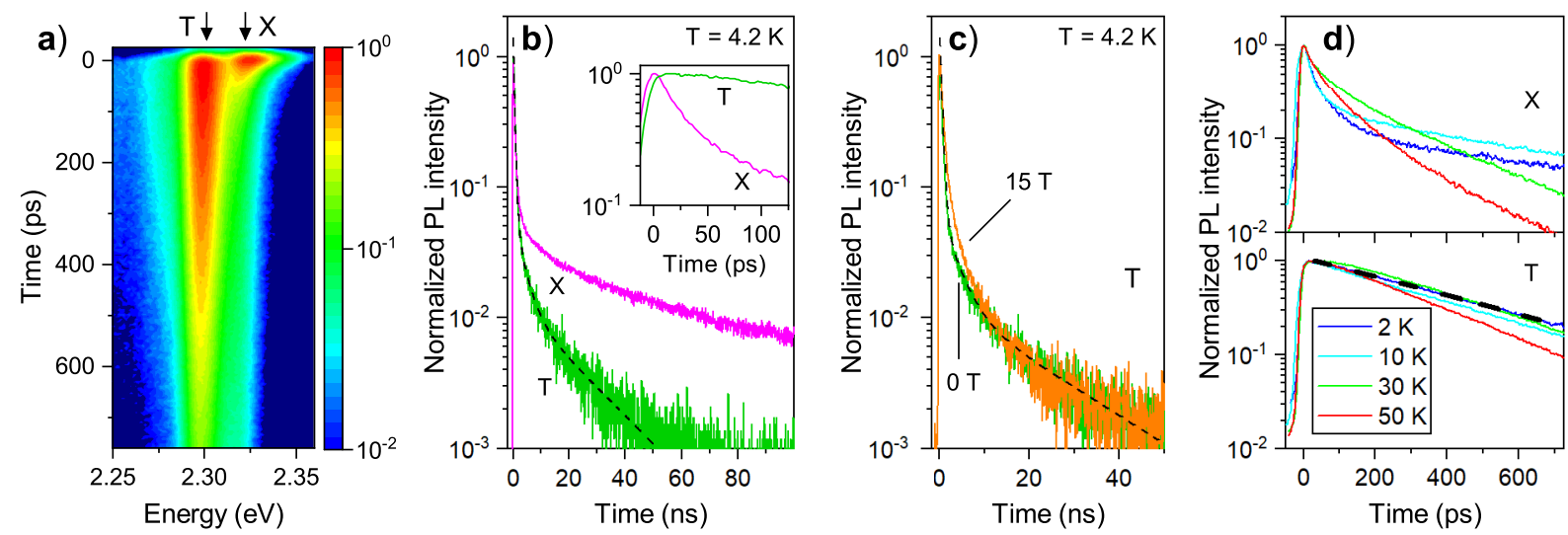

Figure 2: Recombination dynamics. (a) Streak camera image of the photoluminescence of 5ML NPLs at $T=4.2 \mathrm{~K}$. (b) Photoluminescence decays at $T=4.2 \mathrm{~K}$, for the exciton (pink) and lowenergy (green) lines. The dashed line is a three-exponential fit with $\tau_{\mathrm{T}}=450 \mathrm{ps}, \tau_{2}=3 \mathrm{~ns}$, and $\tau_{3}=20 \mathrm{~ns}$. The relative amplitudes of the respective components are $A_{\mathrm{T}}=1, A_{2}=0.04$, and $A_{3}=0.01$. Inset: Initial decays of the exciton and low-energy emission lines, taken from (a). (c) Comparison of the PL decays of the low-energy line at $B=0$ (green) and $15 \mathrm{~T}$ (orange). The dashed line is the same as in panel (b). (d) Normalized PL decays at various temperatures for the excitons (top) and trions (bottom), measured at the line maxima with the streak camera. The dashed line is an exponential fit with $\tau_{\mathrm{T}}=450 \mathrm{ps}$.

sublevels of excitons and trions. For both, the splitting energy is $\Delta E_{\mathrm{Z}}=g \mu_{\mathrm{B}} B$, where $g$ is the $g$-factor, and $\mu_{\mathrm{B}}$ the Bohr magneton. The selection rules for the optical transitions from these spin-split sublevels determine the polarization of the emitted photons. When $k_{\mathrm{B}} T \ll \Delta E_{\mathrm{Z}}$, the lowest Zeeman-split sublevel has a higher occupation and dominates the emission, so that the emission becomes circularly polarized. Note that for the DCP modeling for ensemble of NPLs the $g$-factor anisotropy and various orientation of the NPLs with respect to the external magnetic field direction need to be taken into account. The respective formalism for colloidal nanostructures has been developed in Refs. 10,24,25.

Figure $3 \mathrm{~b}$ shows all possible circularly polarized optical transitions of the trions that are allowed in the electric-dipole approximation, i.e. transitions which change the initial spin by +1 $\left(\sigma^{+}\right.$, red $)$or $-1\left(\sigma^{-}\right.$, blue). Thicker lines indicate transitions from sublevels with higher occupation, which dominate the emission. Importantly, in CdSe the hole and the electron $g$ factors have opposite signs: $g_{\mathrm{h}}<0$ and $g_{\mathrm{e}}>0$, which mandates that the electron with spin $-1 / 2$ and the hole with spin $-3 / 2$ are the low energy states. The negatively charged trions comprise two electrons in a singlet state, therefore the hole $g$-factor alone determines the net Zeeman splitting, so that the lowest sublevel with dominant occupation is $-3 / 2$ (Figure $3 b$, left). The emission from this sublevel is $\sigma^{-}$circularly polarized. The positively charged trions have two holes in a singlet state and therefore the electron $g$-factor alone determines the net Zeeman splitting (Figure 3b, right). The lowest spin sublevel with dominant occupation is $-1 / 2$ and the allowed optical transition is $\sigma^{+}$circularly polarized. Hence, by measuring the DCP sign, $\sigma^{+}$or $\sigma^{-}$, one can unambiguously distinguish between positively and negatively charged trions.

As shown in Figure 3, the negative DCP sign for the low-energy line indicates that it originates from negatively charged trions. Therefore, we conclude that our bare-core CdSe NPLs are partially charged, at least at low temperatures. Similar to CdSe/CdS core/thick-shell $\mathrm{QDs}^{10}$ and CdSe/CdS core/thick-shell NPLs, ${ }^{11}$ this charge is negative.

The magnetic field dependences of the DCP are also very different for excitons and trions. The exciton DCP depends non-monotonically 


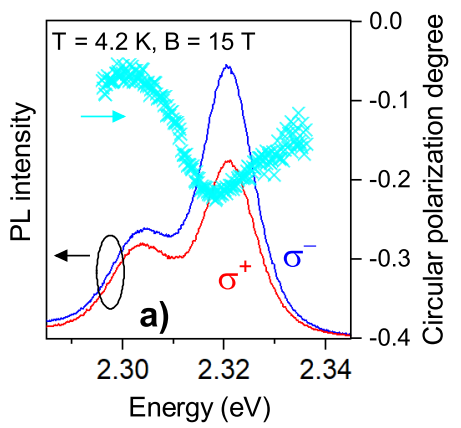

b)
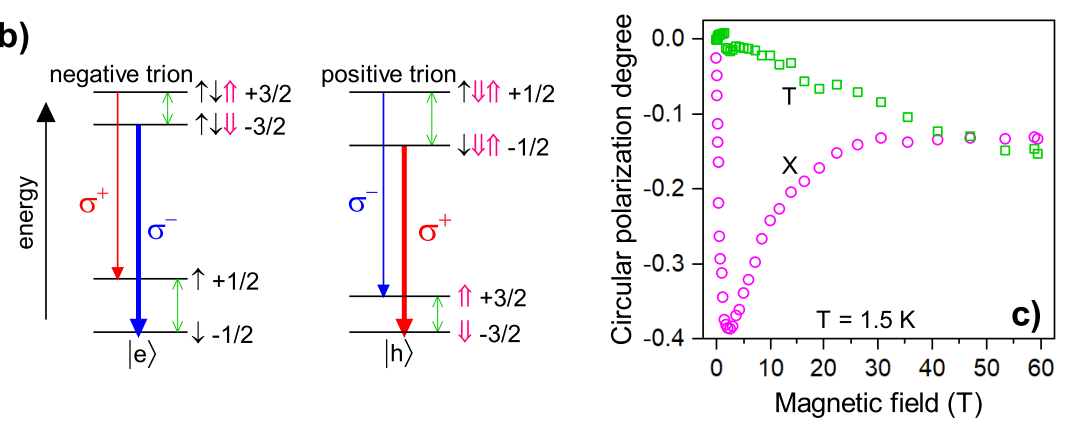

Figure 3: Circular PL polarization in magnetic field. (a) PL spectra of $\sigma^{+}$(red) and $\sigma^{-}$(blue) circular polarization and corresponding DCP (cyan) of 5ML NPLs at $B=15 \mathrm{~T}$ and $T=4.2 \mathrm{~K}$. (b) Schematics of the spin level structure and optical transitions for negatively and positively charged trions in a magnetic field for CdSe-based NPLs with $g_{\mathrm{e}}>0$ and $g_{\mathrm{h}}<0 .{ }^{11}$ The electron and hole Zeeman splittings are shown by the thin green arrows. The short black and pink arrows indicate the electron and hole spins, respectively, and the numbers give the trion spin projections. The polarized optical transitions are shown by the red $\left(\sigma^{+}\right)$and blue $\left(\sigma^{-}\right)$arrows. The more intense emission, shown by the thicker arrows, comes from the lowest-energy trion state with spin $-3 / 2$ for the negative trion, and with spin $-1 / 2$ for the positive trion. (c) Trion and exciton DCP as function of pulsed magnetic field at $T=1.5 \mathrm{~K}$.

on the magnetic field strength (Figure 3c, pink circles). In relatively weak magnetic fields $(B \leq$ $3 \mathrm{~T})$ the DCP value quickly increases from zero at $B=0$ up to $-38 \%$ due to the exchange interaction with surface spins, providing an additional contribution to the exciton Zeeman splitting $E_{\text {exch. }}{ }^{24}$ This interaction increases the Zeeman splitting: $\Delta E_{\mathrm{Z}}^{\mathrm{eff}}=\Delta E_{\mathrm{Z}}+E_{\text {exch }}$, so that the net DCP value becomes larger than the value expected for structures without surface spins. In $B>3 \mathrm{~T}$ the exciton DCP decreases due to the interplay between horizontally and vertically oriented NPL subensembles and saturates at the value of $-13 \%$ in the magnetic field range $30-60$ T. Details of this behavior and its model description are given in Ref. 24, and go beyond the scope of the present paper.

On the contrary, the trion DCP value increases monotonically linear with magnetic field reaching -0.15 in $B=60 \mathrm{~T}$ (Figure 3c, green squares). We conclude that the interaction with the surface spins does not play a role for the negatively charged trions. Two obvious reasons for that are: (i) the two electrons in the trion combine to a singlet state with zero spin, and (ii) due to larger effective mass the hole has smaller wave function density at the NPL surface compared to the electron, and therefore should interact more weakly with surface spins.

Note that the very different polarization properties of the high-energy and low-energy emission lines allow us to exclude LO-phonon assisted exciton recombination and excimer recombination from the possible origins of the low-energy line. The DCP of the LO-phonon assisted exciton recombination is expected to follow the exciton polarization. One can also expect the excimer spin properties to be close to those of the exciton, as the excimer is contributed by the exciton. The role of the surface spins can be different for the excimers from that for the exciton, depending on the surface modification in NPL stacks. But even in the absence of surface spin effects, we would expect the intrinsic Zeeman splitting of an excimer to exhibit a much larger DCP than that of the low-energy line in Figure 3c. Note that in the 5ML NPLs $g_{\mathrm{e}}=1.62 .^{25}$

The hole $g$-factor can be estimated from the magnetic field dependence of the trion DCP by fitting it with $P_{\mathrm{c}}=P_{\mathrm{c}}^{\mathrm{eq}} \tanh \left(\Delta E_{\mathrm{Z}} / 2 k_{\mathrm{B}} T\right)$, where $P_{\mathrm{c}}^{\mathrm{eq}}$ is the DCP saturation value in very strong magnetic fields and $\Delta E_{\mathrm{Z}}=3 g_{\mathrm{h}} \mu_{\mathrm{B}} B$ is the hole Zeeman splitting. ${ }^{11}$ As we did not reach clear DCP saturation in any sample (Fig. S3a), we chose for the evaluation the 
two limiting values of $P_{\mathrm{c}}^{\text {eq }}$ : the largest possible DCP $(-1)$ provides a lower $g_{\mathrm{h}}$ limit, while the highest measured DCP $(-0.15$ in $5 \mathrm{ML})$ provides an upper $g_{\mathrm{h}}$ estimate. The resulting $g_{\mathrm{h}}$ is in the range from -0.03 to -0.2 . These values are in good agreement with theoretical estimations using a parabolic confining potential $\left(g_{\mathrm{h}} \approx-0.1\right),{ }^{11}$ but differ from the value measured in CdSe/CdS NPLs with thick shells $\left(g_{\mathrm{h}} \approx-0.5\right) .{ }^{11}$

Photocharging dynamics of NPLs. Our NPLs exhibit photoinduced modifications of the PL spectra for long-term laser illumination with $10 \mathrm{~mW} / \mathrm{cm}^{2}$. Figure 4a shows PL spectra measured at various times while continuously excited over a total span of 1000 seconds. The exciton PL intensity steadily decreases, while the trion intensity grows. The time evolution of the trion and exciton peak intensities are given in Figure 4b. The strong initial changes saturate after 20 minutes of illumination (open symbols). The exciton fraction in the total PL intensity decreases from $50 \%$ at zero time to $27 \%$ after 2 hours, while the trion fraction increases from $50 \%$ to $73 \%$ (Figure S4). It is natural to assign this effect to the NPL photocharging, as the trion emission is due to singly-charged NPLs. The photocharging is very stable at low temperatures, and persists in darkness for at least 5 hours. The closed symbols in Figure 4b are measured for the sample kept without illumination except for the very short measurement times for recording the PL when the laser is unblocked. However, heating the sample up to room temperature erases the photocharging. When the sample is cooled down again to $T=4.2 \mathrm{~K}$, the exciton and trion intensities are restored, as one can see from the data points at 6.2 hours. The sum of the PL intensities of the exciton and trion lines is also sensitive to photocharging. It decreases by about $15 \%$ under illumination, but recovers after warming to room temperature (Figure 4c). The nearly complete recovery indicates that the observed changes are not caused by sample degradation under illumination.

Trion binding energy. The energy separation between the exciton and trion lines can be interpreted as the trion binding energy $E_{\mathrm{b}}^{\mathrm{T}}$.
As one can see in Figure 1c, it increases from $18 \mathrm{meV}$ to $30 \mathrm{meV}$ with reducing the NPL thickness from $5 \mathrm{ML}$ down to 3ML. This is the expected behavior for charged excitons, for which the binding energy increases with reducing the dimensionality from 3D towards 2D. Importantly, the shifts between the exciton and trion lines do not match the LO phonon energy of $25.4 \mathrm{meV}^{22,30}$ (see dashed line in Figure 1c). This rules out the assignment of the low-energy line to an LO-phonon assisted exciton recombination mechanism.

It is instructive to compare the trion binding energy in CdSe NPLs with that in epitaxiallygrown quantum wells (QWs), where trions and excitons can also be considered as quasi-twodimensional. There is no reliable information on trions in CdSe-based QWs. However, it was shown in Ref. 31 that the trion binding energy in QWs based on various other semiconductors including GaAs, CdTe, and ZnSe, shows a universal behavior when it is normalized to the bulk exciton Rydberg energy, $R y$, of the corresponding materials. In that paper experimental results for QWs of different widths were collected and compared with model calculations. In the quasi-2D limit of very thin QWs the exciton binding energy $E_{\mathrm{b}}^{\mathrm{X}}=4 R y$ and the trion binding energy $E_{\mathrm{b}}^{\mathrm{T}}=0.48 R y$, which results in $E_{\mathrm{b}}^{\mathrm{T}} / E_{\mathrm{b}}^{\mathrm{X}}=0.12 .{ }^{31,32}$ This situation is obviously an idealization as the Coulomb interaction is always acting in three dimensions, leading to a reduced enhancement of the binding energies. Moreover, in these epitaxial structures, where the QW is embedded from both sides in semiconductor material, dielectric enhancement is typically neglected, as the dielectric constants of QW and barriers are assumed to be homogeneous. It becomes relevant, however, when the QW is moved towards the surface. ${ }^{33}$

In bulk CdSe $R y=15 \mathrm{meV} .{ }^{34}$ By normalizing the trion binding energy in our NPLs to this value we get $E_{\mathrm{b}}^{\mathrm{T}} / R y=1.2-2$, which is up to 4 times larger than in the epitaxial QWs. Such an increase originates from the dielectric enhancement effect for exciton complexes. ${ }^{20,35} \mathrm{Di}$ electric enhancement occurs in structures where the excitons are localized in a material with large dielectric constant, surrounded by a ma- 

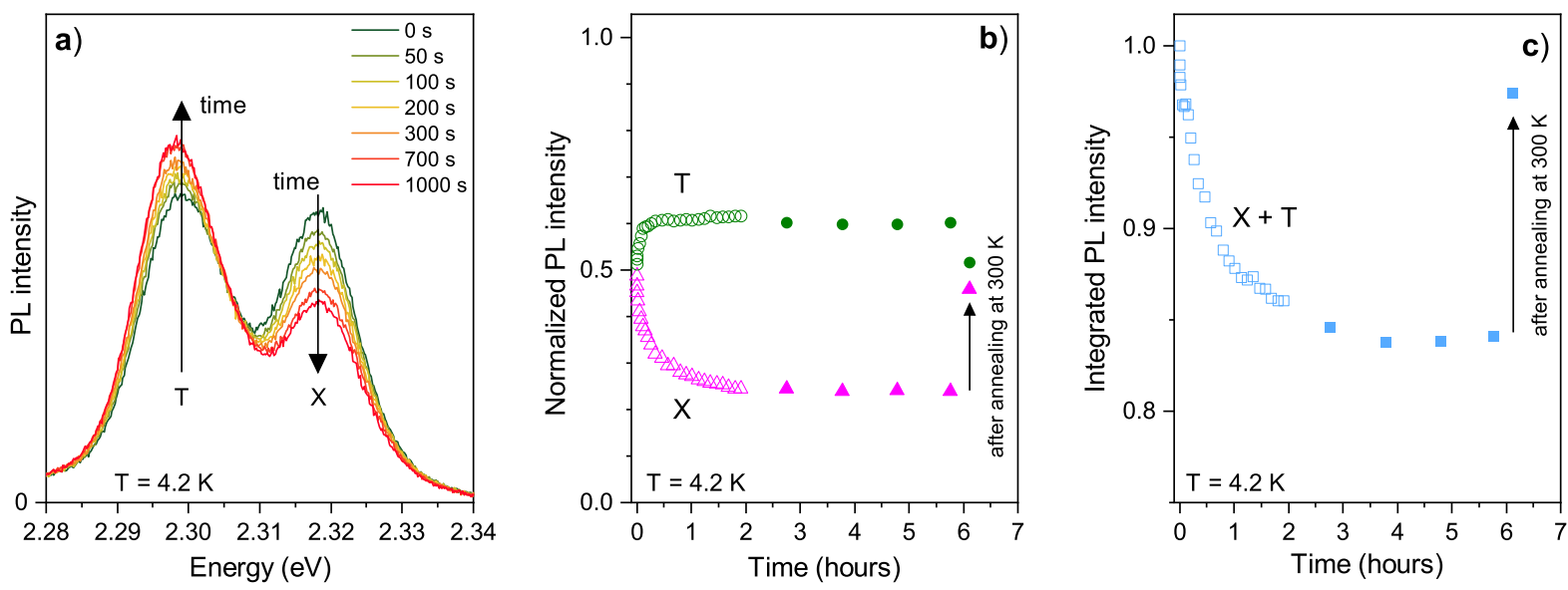

Figure 4: PL evolution of the 5ML NPLs under illumination with laser power of $10 \mathrm{~mW} / \mathrm{cm}^{2}$ at $T=4.2 \mathrm{~K}$. (a) PL spectra measured under continuous illumination over the course of $1000 \mathrm{~s}$ (from green to red). The spectrum acquisition time is $1 \mathrm{~s}$. The exciton intensity decreases, while the trion intensity increases. (b) Normalized peak intensity of exciton and trion lines over illumination time. (c) Temporal evolution of the total spectrally-integrated PL intensity. In panels (b,c) open symbols are measured under continuous illumination, closed symbols are for the sample kept in darkness except for the short PL acquisition time. The last data points at 6.2 hours are acquired after the sample is warmed to room temperature and cooled again to $4.2 \mathrm{~K}$. This temperature treatment erases the photocharging effect.

terial with considerably smaller dielectric constant. Our NPLs are composed of CdSe with a dielectric constant of 6 (the high-frequency value) to 8.4 (the low-frequency value). ${ }^{20}$ The dielectric constant of the surrounding organic ligands is about 2 . The dielectric enhancement effect is taken into account by the attraction of the exciton electron to a hole image charge, and of the exciton hole to an electron image charge. ${ }^{36,37}$ For the studied CdSe NPLs we have measured exciton binding energies of $160-200 \mathrm{meV}$ for $5-3 \mathrm{ML}$ NPLs (details will be published elsewhere), which agrees well with the theoretically estimated values, ${ }^{38}$ and is about 3 times larger than the ideal $2 \mathrm{D}$ limit of the QW case $4 R y=60 \mathrm{meV}$. Interestingly, the ratio of the trion to exciton binding energy is only weakly affected by the dielectric enhancement effect and remains in the range $E_{\mathrm{b}}^{\mathrm{T}} / E_{\mathrm{b}}^{\mathrm{X}}=0.11-0.15$. We conclude from the above considerations that both the exciton and trion binding energies in NPLs are drastically enhanced (about 3-4 times compared to the 2D QW limit) due to dielectric enhancement.

In summary, we used several experimental approaches to uniquely identify the origin of the low-energy emission line in CdSe nanoplatelets as the emission from negatively charged excitons (trions). The recombination dynamics and its changes with temperature and magnetic field, spectral changes, and spin polarization induced by a strong magnetic field contributed to the identification. The trion binding energy varies from $18 \mathrm{meV}$ in $5 \mathrm{ML}$ NPLs to $30 \mathrm{meV}$ in 3ML samples; its large value is to a great extent provided by dielectric enhancement.

\section{Methods}

Samples. Bare-core CdSe colloidal nanoplatelets with thicknesses of 3, 4 and 5 monolayers were synthesized according to the protocol reported in Ref. 1. They consist of several (3 to 5) CdSe monolayers plus an additional Cd layer, so that both NPL surfaces are Cd-terminated. The detailed characterization of the samples is given in Ref. 20. The samples studied here are from the same batches as in Refs 20 and 25 (identical sample labels) and Ref. 24 (Sample 1 corresponds to $4 \mathrm{ML}$, and Sample 4 to $5 \mathrm{ML}$ ). For low-temperature measurements the NPLs 
were drop-cast onto quartz plates.

Absorption measurements. For the absorption spectra measurements at $T=4.2 \mathrm{~K}$ the samples were immersed in a liquid helium bath optical cryostat and illuminated by an incandescent lamp with a broad spectrum. The transmitted light was dispersed by means of a 0.55-m spectrometer and detected by a liquidnitrogen-cooled charge-coupled-device (CCD) camera.

Photoluminescence excitation (PLE) spectra measurements. For the PLE spectra measurements at $T=4.2 \mathrm{~K}$ the samples were excited with laser pulses with a pulse duration of $\sim 2.5 \mathrm{ps}$, a linewidth of $\sim 0.23$ $\mathrm{nm}$, a repetition frequency $30 \mathrm{kHz}$, and an average excitation density $100 \mathrm{~mW} / \mathrm{cm}^{2}$. The laser wavelength was tuned across the corresponding exciton transitions. To reduce the scattered laser light, the PL was filtered with a corresponding band-pass filter then with a 0.55 $\mathrm{m}$ spectrometer, before being detected with a CCD at the low-energy tails of the spectra, e.g. at $2.28 \mathrm{eV}$ in $5 \mathrm{ML}$ NPLs.

Magneto-optical measurements in dc magnetic fields. The samples were mounted in a titanium sample holder on top of a threeaxis piezo-positioner and placed in the variable temperature insert $(2.2-70 \mathrm{~K})$ of a liquid helium bath cryostat equipped with a superconducting solenoid (magnetic fields up to $15 \mathrm{~T}$ ). The measurements were performed in the Faraday geometry (light excitation and detection parallel to the magnetic field direction). The photoluminescence was excited using a pulsed diode laser (photon energy $3.06 \mathrm{eV}$, wavelength $405 \mathrm{~nm}$, pulse duration $50 \mathrm{ps}$, pulse repetition rate between 0.8 and $5 \mathrm{MHz}$ ) with a weak average excitation density of $10 \mathrm{~mW} / \mathrm{cm}^{2}$. The $\mathrm{PL}$ was detected in backscattering geometry, dispersed with a $0.55-\mathrm{m}$ spectrometer and measured either by a liquid-nitrogen-cooled CCD camera or a $\mathrm{Si}$ avalanche photodiode. The PL circular polarization degree was analyzed by a combination of a quarter-wave plate and a linear polarizer.

Magneto-optical measurements in pulsed magnetic fields. The samples were mounted on a custom fiber-coupled probe in a helium bath cryostat with a long tail extending into the bore of a $65 \mathrm{~T}$ pulsed magnet. The experiments were performed at $T=1.5 \mathrm{~K}$. The pump laser light was directed to the sample using a $600-\mu \mathrm{m}$ diameter multimode optical fiber; the same fiber was used to collect the emitted PL. Thin film circular polarizers were used to select $\sigma^{-}$and $\sigma^{+}$polarized PL. Full optical spectra were acquired every $1 \mathrm{~ms}$ continuously throughout the magnet pulse using a fast CCD camera. To switch between $\sigma^{-}$and $\sigma^{+}$polarization, we switched the direction of the current in the magnet.

Time-resolved measurements with an avalanche photodiode. The samples were excited with a pulsed diode laser (see above). The PL decay was measured with a Si avalanche photodiode connected to a conventional timecorrelated single-photon counting setup. The instrumental response time was about $200 \mathrm{ps}$.

Time-resolved measurements with a streak camera. The NPLs were excited by a frequency-doubled mode-locked Ti-Sapphire laser (photon energy $3.06 \mathrm{eV}$, wavelength $405 \mathrm{~nm}$, pulse duration $2 \mathrm{ps}$, repetition rate $76 \mathrm{MHz}$ ). Time-resolved PL spectra were recorded by a streak camera attached to a $0.5-\mathrm{m}$ spectrometer, providing temporal and spectral resolution of $\lesssim 5 \mathrm{ps}$ and $\lesssim 1 \mathrm{~nm}$. The samples were immersed in the variable temperature insert of a bath cryostat $(2-60 \mathrm{~K}$ temperature range), the average excitation power was $10 \mathrm{~mW} / \mathrm{cm}^{2}$.

\section{ASSOCIATED CONTENT}

Supporting Information. PL, PLE and absorption spectra of all studied CdSe NPLs, their recombination dynamics and spin polarization induced by a magnetic field. Additional information about the photocharging changes of exciton and trion PL.

\section{AUTHOR INFORMATION}

Corresponding Authors:

Email: elena.shornikova@tu-dortmund.de

Email: dmitri.yakovlev@tu-dortmund.de

\section{ORCID}

Elena V. Shornikova: 0000-0002-6866-9013

Dmitri R. Yakovlev: 0000-0001-7349-2745 
Louis Biadala: 0000-0002-1953-9095

Scott A. Crooker: 0000-0001-7553-4718

Vasilii V. Belykh: 0000-0002-0032-748X

Mikhail V. Kochiev: 0000-0002-5653-6416

Michel Nasilowski: 0000-0002-2484-7674

Benoit Dubertret: 0000-0002-9450-8029

Manfred Bayer: 0000-0002-0893-5949

\section{Notes}

The authors declare no competing financial interests.

\section{Acknowledgements}

The authors are thankful to A. V. Rodina, A. A. Golovatenko, and Al. L. Efros for fruitful discussions. We acknowledge support by the Deutsche Forschungsgemeinschaft via the International Collaborative Research Centre TRR 160 (Project B1). Work at the NHMFL is supported by the National Science Foundation DMR-1644779, the State of Florida, and the U.S. Department of Energy. D.R.Y. acknowledges the partial support of the Russian Science Foundation (Project No. 20-42-01008). V.V.B. and M.V.K. acknowledge the support of the Russian Foundation for Basic Research (Project No. 18-32-20202). L.B. acknowledges the support from Agence Nationale de la Recherche (Grant No. TROPICAL ANR-19-CE09-002201).

\section{References}

1. Ithurria, S.; Dubertret, B. Quasi 2D colloidal CdSe platelets with thicknesses controlled at the atomic level. J. Am. Chem. Soc. 2008, 130, 16504-16505.

2. Nasilowski, M.; Mahler, B.; Lhuillier, E.; Ithurria, S.; Dubertret, B. Two-dimensional colloidal nanocrystals. Chem. Rev. 2016, 116, 10934-10982.

3. Ithurria, S.; Tessier, M. D.; Mahler, B.; Lobo, R. P. S. M.; Dubertret, B.; Efros, Al. L. Colloidal nanoplatelets with two-dimensional electronic structure. Nat. Mater. 2011, 10, 936-941.
4. Feng, D.; Yakovlev, D. R.; Pavlov, V. V.; Rodina, A. V.; Shornikova, E. V.; Mund, J.; Bayer, M. Dynamic evolution from negative to positive photocharging in colloidal CdS quantum dots. Nano Lett. 2017, 17, 2844 2851.

5. Hu, R.; Wu, Z.; Zhang, Y.; Yakovlev, D. R.; Liang, P.; Qiang, G.; Guo, J.; Jia, T.; Sun, Z.; Bayer, M.; Feng, D. H. Long-lived negative photocharging in colloidal CdSe quantum dots revealed by coherent electron spin precession. J. Phys. Chem. Lett. 2019, 10, 4994-4999.

6. Hu, R.; Yakovlev, D. R.; Liang, P.; Qiang, G.; Chen, C.; Jia, T.; Sun, Z.; Bayer, M.; Feng, D. H. Origin of two Larmor frequencies in the coherent spin dynamics of colloidal CdSe quantum dots revealed by controlled charging. J. Phys. Chem. Lett. 2019, 10, 3681-3687.

7. Kheng, K.; Cox, R. T.; d'Aubigné, M. Y.; Bassani, F.; Saminadayar, K.; Tatarenko, S. Observation of negatively charged excitons $X^{-}$in semiconductor quantum wells. Phys. Rev. Lett. 1993, 71, 1752-1755.

8. Efros, Al. L.; Nesbitt, D. J. Origin and control of blinking in quantum dots. Nat. Nanotechnol. 2016, 11, 661-671.

9. Galland, C.; Ghosh, Y.; Steinbrück, A.; Hollingsworth, J. A.; Htoon, H.; Klimov, V. I. Lifetime blinking in nonblinking nanocrystal quantum dots. Nat. Commun. 2012, 3, 908.

10. Liu, F.; Biadala, L.; Rodina, A. V.; Yakovlev, D. R.; Dunker, D.; Javaux, C.; Hermier, J. P.; Efros, Al. L.; Dubertret, B.; Bayer, M. Spin dynamics of negatively charged excitons in $\mathrm{CdSe} / \mathrm{CdS}$ colloidal nanocrystals. Phys. Rev. $B$ 2013, 88, 035302 .

11. Shornikova, E. V.; Biadala, L.; Yakovlev, D. R.; Feng, D.; Sapega, V. F.; 
Flipo, N.; Golovatenko, A. A.; Semina, M. A.; Rodina, A. V.; Mitioglu, A. A.; Ballottin, M. V.; Christianen, P. C. M.; Kusrayev, Yu. G.; Nasilowski, M.; Dubertret, B.; Bayer, M. Electron and hole g-factors and spin dynamics of negatively charged excitons in $\mathrm{CdSe} / \mathrm{CdS}$ colloidal nanoplatelets with thick shells. Nano Lett. 2018, 18, 373-380.

12. Antolinez, F. V; Rabouw, F. T.; Rossinelli, A. A.; Cui, J.; Norris, D. J. Observation of electron shake-up in CdSe/CdS core/shell nanoplatelets. Nano Lett. 2019, 19, 8495-8502.

13. Pinchetti, V.; Shornikova, E. V.; Qiang, G.; Bae, W. K.; Crooker, S. A.; Yakovlev, D. R.; Bayer, M.; Klimov, V. I.; Brovelli, S. Dual-emitting dot-in-bulk CdSe/CdS nanocrystals with highly emissive core- and shell-based trions sharing the same resident electron. Nano Lett. 2019, 19, 8846--8854.

14. Javaux, C.; Mahler, B.; Dubertret, B.; Shabaev, A.; Rodina, A. V.; Efros, Al. L.; Yakovlev, D. R.; Liu, F.; Bayer, M.; Camps, G.; Biadala, L.; Buil, S.; Quelin, $\mathrm{X}$; Hermier, J-P. Thermal activation of non-radiative Auger recombination in charged colloidal nanocrystals. Nat. Nanotechnol. 2013, 8, 206-212.

15. Bae, W. K.; Padilha, L. A.; Park, Y.-S.; McDaniel, H.; Robel, I.; Pietryga, J. M.; Klimov, V. I. Controlled alloying of the core-shell interface in CdSe/CdS quantum dots for suppression of Auger recombination. ACS Nano 2013, \%, 3411-3419.

16. Park, Y.-S.; Bae, W. K.; Padilha, L. A.; Pietryga, J. M.; Klimov, V. I. Effect of the core/shell interface on Auger recombination evaluated by single-quantum-dot spectroscopy. Nano Lett. 2014, 14, 396-402.

17. Nasilowski, M.; Spinicelli, P.; Patriarche, G.; Dubertret, B. Gradient CdSe/CdS quantum dots with room temperature biexciton unity quantum yield. Nano Lett. 2015, 15, 3953-3958.
18. Pelton, M.; Andrews, J. J.; Fedin, I.; Talapin, D. V.; Leng, H.; O'Leary, S. K. Nonmonotonic dependence of Auger recombination rate on shell thickness for $\mathrm{CdSe} / \mathrm{CdS}$ core/shell nanoplatelets. Nano Lett. 2017, 17, 6900-6906.

19. Biadala, L.; Liu, F.; Tessier, M. D.; Yakovlev, D. R.; Dubertret, B; Bayer, M. Recombination dynamics of band edge excitons in quasi-two-dimensional CdSe nanoplatelets. Nano Lett. 2014, 14, 11341139.

20. Shornikova, E. V.; Biadala, L.; Yakovlev, D. R.; Sapega, V. F.; Kusraev, Yu. G.; Mitioglu, A. A.; Ballottin, M. V.; Christianen, P. C. M.; Belykh, V. V.; Kochiev, M. V.; Sibeldin, N. N.; Golovatenko, A. A.; Rodina, A. V.; Gippius, N. A.; Kuntzmann, A.; Jiang, Y.; Nasilowski, M.; Dubertret, B.; Bayer, M. Addressing the exciton fine structure in colloidal nanocrystals: the case of CdSe nanoplatelets. Nanoscale 2018, 10, 646-656.

21. Tessier, M. D.; Biadala, L.; Bouet, C.; Ithurria, S.; Abecassis, B.; Dubertret, B. Phonon line emission revealed by selfassembly of colloidal nanoplatelets. ACS Nano 2013, 7, 3332-3340.

22. Diroll, B. T.; Cho, W.; Coropceanu, I.; Harvey, S. M.; Brumberg, A.; Holtgrewe, N.; Crooker, S. A.; Wasielewski, M. R.; Prakapenka, V. B.; Talapin, D. V.; Schaller, R. D. Semiconductor nanoplatelet excimers. Nano Lett. 2018, 18, 6948-6953.

23. Achtstein, A. W.; Scott, R.; Kickhöfel, S.; Jagsch, S. T.; Christodoulou, S.; Bertrand, G. H. V.; Prudnikau, A. V.; Antanovich, A.; Artemyev, M.; Moreels, I.; Schliwa, A.; Woggon, U. p-State luminescence in CdSe nanoplatelets: Role of lateral confinement and a longitudinal optical phonon bottleneck. Phys. Rev. Lett. 2016, 116, 116802. 
24. Shornikova, E. V.; Golovatenko, A. A.; Yakovlev, D. R.; Rodina, A. V.; Biadala, L.; Qiang, G.; Kuntzmann, A.; Nasilowski, M.; Dubertet, B.; Polovitsyn, A.; Moreels, I.; Bayer, M. Surface spin magnetism controls the polarized exciton emission from CdSe nanoplatelets. Nat. Nanotechnol. 2020, https://doi.org/10.1038/s41565-019-06317

25. Kudlacik, D.; Sapega, V. F.; Yakovlev, D. R.; Kalitukha, I. V.; Shornikova, E. V.; Rodina, A. V.; Ivchenko, E. L.; Dimitriev, G. S.; Nasilowski, M.; Dubertet, B.; Bayer, M. Single and double electron spin-flip Raman scattering in CdSe colloidal nanoplatelets. Nano Lett. 2020, 20, 517-525.

26. Louyer, Y.; Biadala, L.; Tamarat, Ph.; Lounis, B. Spectroscopy of neutral and charged exciton states in single CdSe/ZnS nanocrystals. Appl. Phys. Lett. 2010, 96, 203111.

27. Fernee, M. J.; Littleton, B. N.; Rubinsztein-Dunlop, H. Detection of bright trion states using the fine structure emission of single $\mathrm{CdSe} / \mathrm{ZnS}$ colloidal quantum dots. ACS Nano 2009, 3, 3762-3768.

28. Fernee, M. J.; Sinito, C.; Louyer, Y.; Potzner, C.; Nguyen, T.-L.; Mulvaney, P.; Tamarat, P.; Lounis, B. Magneto-optical properties of trions in non-blinking charged nanocrystals reveal an acoustic phonon bottleneck. Nat. Commun. 2012, 3, 1287.

29. Califano, M.; Franceschetti, A.; Zunger, A. Lifetime and polarization of the radiative decay of excitons, biexcitons, and trions in CdSe nanocrystal quantum dots. Phys. Rev. B 2007, 75, 115401.

30. Cherevkov, S. A.; Fedorov, A. V.; Artemyev, M. V.; Prudnikau, A. V.; Baranov, A. V. Anisotropy of electron-phonon interaction in nanoscale CdSe platelets as seen via off-resonant and resonant Raman spectroscopy. Phys. Rev. B 2013, 88, 041303(R).

31. Sergeev, R. A.; Suris, R. A.; Astakhov, G. V.; Ossau, W.; Yakovlev, D. R. Universal estimation of $\mathrm{X}^{-}$trion binding energy in semiconductor quantum wells. Eur. Phys. J. B 2005, 47, 541-547.

32. Courtade, E.; Semina, M.; Manca, M.; Glazov, M. M.; Robert, C.; Cadiz, F.; Wang, G.; Taniguchi, T.; Watanabe, K.; Pierre, M.; Escoffier, W.; Ivchenko, E. L.; Renucci, P.; Marie, X.; Amand, T.; Urbaszek, B. Charged excitons in monolayer $\mathrm{WSe}_{2}$ : experiment and theory. Phys. Rev. B 2017, 96, 085302 .

33. Kulik, L. V.; Kulakovskii, V. D.; Bayer, M.; Forchel, A.; Gippius, N. A.; Tikhodeev, S.G. Dielectric enhancement of excitons in near-surface quantum wells. Phys. Rev. B 1996, 54, 2335-2338(R).

34. Voigt, J.; Spiegelberg, F.; Senoner, M. Band parameters of CdS and CdSe single crystals determined from optical exciton spectra. Phys. Stat. Sol. B 1979, 91, 189-199.

35. Gippius, N. A.; Yablonskii, A. L.; Dzyubenko, A. B.; Tikhodeev, S. G.; Kulik, L. V.; Kulakovskii, V. D.; Forchel, A. Excitons in near-surface quantum wells in magnetic fields: Experiment and theory. J. Appl. Phys. 1998, 83, 5410-5417.

36. Rytova, N. S. Coulomb interaction of electrons in a thin film. Dokl. Akad. Nauk SSSR 1965, 163, 1118-1120. [Sov. Phys. Dokl. 1965, 10, 754-756.]

37. Keldysh, L. V. Coulomb interaction in thin semiconductor and semimetal films. JETP Lett. 1979, 29, 658-660.

38. Benchamekh, R.; Gippius, N. A.;Even, J.; Nestoklon, M. O.; Jancu, J.-M.; Ithurria, S.; Dubertret, ; Efros, Al. L.; Voisin, P. Tight-binding calculations of image-charge 
effects in colloidal nanoscale platelets of CdSe. Phys. Rev. B 2014, 89, 035307. 